\title{
MIMO Channel Modeling and Simulation
}

\author{
R. Parra-Michel, A. Alcocer-Ochoa, \\ A. Sanchez-Hernandez and Valeri Kontorovich \\ CINVESTAV-IPN \\ Mexico
}

\section{Introduction}

New communications standards are continuously appearing as response to the increasing demands of users and telecommunication applications. Those demands reflect requirements regarding raising the data rates and user's mobility. During these years we shall witness an introduction of applications for indoor wireless networks at the $60 \mathrm{GHz}$ band, Vehicle to Vehicle wireless systems, mobile Internet and VoIP over WiMax based systems, etc. Those new applications will rely on Multiple Input and Multiple-Output (MIMO) systems for being able to handle concepts of radar, beamforming, and spatial multiplexing.

In such variety of applications where all of them uses radio waves to guarantee the mobility requirement, plenty of radio propagation channels will be undoubtedly utilized. It is then mandatory to count with channel models that adequately describe the propagation channel by means of some mathematical expressions. Thus, the communication researcher can devise algorithms to counteract the distortions introduced by the channel.

Once mathematical models for channels and algorithms for the communication blocks are conceived, the next step in communication systems design flow is to prove whether the proposed solution attains the desired functionality.

Notwithstanding, there are several factors intervening in these designs; e.g., the multiple choices for communication processing algorithms, the large variety of channel environments with their random nature, the inherent computational complexity of each communications algorithms, etc. These factors preclude obtaining closed form expressions for the entire system performance. One must thus rely on simulations. These simulations provide a system's performance at various design stages, while allowing design engineers to test several arrangements with different algorithms over several channel models, until the final architecture matches the requested performance.

The term simulation hereafter is referred to a performance analysis by means of computer programs. Another close related term is emulation, which refers to tests of the implemented system on digital circuits, such as Complex Programmable Logic Devices (CPLD) and Field Programmable Gate Arrays (FPGA). Emulation also avoids the final construction of a system under design; in fact, emulation has gotten to be a necessity for the newest communication systems, whose throughput is high enough to preclude simulation by computer programs. Even though simulation models are actually computer programs and 
emulators are Hardware (HW) devices, we will refer to both of them hereafter by the single term of simulation.

But counting with simulations results still remains open the question whether the approved design will actually work as expected in the real implementation or not. The probability of success highly depends on how close the channel model resembles the real channel conditions, i.e. its ability to distort the signals in the same (statistically) manner than the real channel performs. The failing of good channel models will yield wrong results of system performance that can lead to system redesign, an incident that have happened in the development of initial standards (Woerner et al., 1994). Thus, strong efforts are devoted to obtain good channel models and its efficient implementations as channel simulators.

In this Chapter it will be discussed how to construct channel simulators for MIMO wireless systems. Considering that the channel is an artificial construction that can integrate the propagation media and several blocks of the communication system, we can obtain several definitions for channels. Here we consider the channel simulators that deal with the analogic channel; i.e. simulators for testing physical layer algorithms such as channel estimation.

The Chapter is organized as follows: The general problem of channel modeling is discussed in Section 2. Within this section, the main propagation phenomena of the radio channel and its deterministic mathematical expressions are devised. These descriptions are then analyzed in statistical terms to define the final radio channel expressions. The propagation media is then included in a MIMO System and the MIMO channel model is finally established. This Section ends with the delimitation of the channels to be discussed throughout this Chapter.

In Section 3 two main approaches that try to explain and/or replicate the propagation phenomena are presented. These models are the geometrical based approach and the method of artificial paths. Proposed simulators according to the commented modeling approaches are discussed in Section 4. This Section also provides a brief space of comments regarding the practical implementation issues. Section 5 defines a metric that permits the performance comparison of the proposed simulators along with simulation results. Section 6 is devoted to point out the open research areas and future trends in this topic. The Chapter ends with Section 7, where the conclusions of the manuscript are drawn. Finally, it is worth to mention here that this chapter does not try to enlist all the models and simulators for wireless channels, but to present the main ideas of the topic, connect all the details, and present the recent advances in this area of research.

\section{The radio channel model}

At the frequencies prescribed by communication standards, radio signals suffer the effects of attenuation, diffraction, refraction and reflection, with the last three causing multipath propagation. This means that several replicas of the transmitted signal will arrive at the receiver through different paths. Signals along each path will experience variations in amplitude and delay; consequently the composite signal at the receiver becomes distorted.

Due to the complexity involved in propagation analysis, all of the amplitude and delay variations are treated as random, as well as time variant features. A simplified example of multipath propagation is provided by Fig. 1, with small number of paths involved. It is considered in this figure that a signal departs from an array of $\mathrm{N}$ antennas at the 
transmitter's side ( $\mathrm{Tx}$ ) and arrives to an antenna array of $\mathrm{M}$ elements at the receiver's side $(\mathrm{Rx})$. For the figure $\mathrm{N}=\mathrm{M}=3$, and there are three main paths (dp1 to dp3) departing from $\mathrm{Tx}$ and four paths (ap1 to ap4) arriving at Rx.

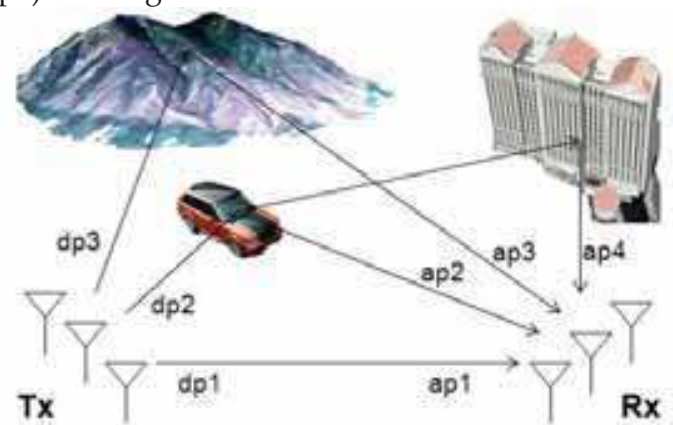

Fig. 1. Multipath propagation in a MIMO wireless link.

The following issues, concerning multipath propagation, become evident in Fig. 1:

1. Signals from each path can arrive at $\mathrm{Rx}$ with different azimuth and elevation angles.

2. From all the energy being transmitted, only a fraction of it will effectively reach the receiver. This can be interpreted as the transmitter scattering the energy over several paths, each one with a different angle of departure.

3. As some paths are longer than others (see path dp3-ap3), the different replicas will arrive with different time delays $(\tau)$ with respect to the first incoming signal (in this case, see dp1-ap1).

4. When scatters in the propagation media or the receiver are in movement, the signals will experience a Doppler frequency shift $(v)$ with respect to the carrier frequency. In the figure, the paths connected with dp2 will present this effect.

The above issues concerning multipath propagation are treated in detail in (Parsons, 2000).

\subsection{Deterministic description of the MIMO Radio Channel}

In what follows, adequate mathematical descriptions that comprise the main propagation phenomena are constructed. It has been, however, discarded the discussion concerning link budget and shadowing, but it will be centered on the effects of scattering.

Define a time dependent signal $x(t)$ transmitted nearby a point in the (three dimensional) space $\boldsymbol{\rho}^{\mathrm{Tx}}$, hereafter defined as the transmitter reference point, that modulates a carrier wave with central radian frequency $\omega_{c}$, expressed as $\cos \left(\omega_{c} t\right)$. A received signal $y(t)$ can be measured in a point nearby a receiver reference point $\boldsymbol{\rho}^{\mathrm{Rx}}$. The received signal can be expressed as:

$$
y(t)=\sum_{l=1}^{L_{p}(t)} A_{l}(t) x\left(t-d_{l}(t)\right) \cos \left(\omega_{c}\left(t-d_{l}(t)\right)-\theta_{l}^{\mathrm{Tx}}-\theta_{l}^{\mathrm{Rx}}\right) .
$$

The last equation considers a single input and single output system (SISO), that according to the propagation phenomena, $l=1,2, \ldots, L_{p}(t)$ paths with replicas of the transmitted signals 
will contribute with energy to the measurement point. Each path will arrive with a time dependent delay $d_{l}(t)$ with respect to the first incoming path, and with time varying amplitudes $A_{l}(t)$. Phases $\theta_{l}^{\mathrm{Tx}}$ and $\theta_{l}^{\mathrm{Rx}}$ correspond to the difference of phases between the $l$ path and a reference wave transmitted from $\boldsymbol{\rho}^{\mathrm{Tx}}$ and arriving at $\boldsymbol{\rho}^{\mathrm{Rx}}$, respectively.

Supposing that in a block of transmitted data, amplitudes $A_{l}(t)$ can be relaxed as constant parameter $A_{l}$, and that the number of paths remains constant (but relatively large) and is equal to $L_{p}$. Besides this, $d_{l}(t)$ is expressed as a linear function of $t$, it means, $d_{l}(t)=\tau_{l}+\varsigma_{l} t$, where $\tau_{l}$ is a fixed delay and $\varsigma_{l}$ is the rate of change of the delay of the $l$-path. With the commented assumptions, equation (1) can be written as:

$$
y(t)=\sum_{l=1}^{L_{p}} A_{l} x\left(t-\tau_{l}-\varsigma_{l} t\right) \cos \left(\omega_{c}\left(t-\tau_{l}-\varsigma_{l} t\right)-\theta_{l}^{\mathrm{Tx}}-\theta_{l}^{\mathrm{Rx}}\right) .
$$

Considering now that the rate of change of the transmitted signal is much more bigger than every rate of change of the paths delays, $x\left(t-\tau_{l}-\varsigma_{l} t\right)$ can be fairly good approximated by $x\left(t-\tau_{l}\right) ; v_{l}$ is $\omega_{c} \varsigma_{l}$, the Doppler shift of the $l$-path and $\theta_{l}^{c}=\omega_{c} \tau_{l}$ is an unknown carrier phase. The former considerations are explained in detail in (Kennedy, 1969). Defining the points in the space $\boldsymbol{\rho}_{1}^{\mathrm{Tx}}$ and $\boldsymbol{\rho}_{1}^{\mathrm{Rx}}$ as the true positions of the transceivers, the received signal can be expressed as:

$$
y(t)=\sum_{l=1}^{L_{p}} A_{l} x\left(t-\tau_{l}\right) \cos \left(\left(\omega_{c}-v_{l}\right) t-\theta_{l}^{c}-\frac{2 \pi}{\lambda} \mathbf{k}_{l}^{\mathrm{Tx}} \cdot \boldsymbol{\rho}_{1}^{\mathrm{Tx}}-\frac{2 \pi}{\lambda} \mathbf{k}_{l}^{\mathrm{Rx}} \cdot \boldsymbol{\rho}_{1}^{\mathrm{Rx}}\right),
$$

where $\mathbf{k}_{l}^{\mathrm{Tx}}$ and $\mathbf{k}_{l}^{\mathrm{Rx}}$ are unitary three dimensional vectors pointing at the angle of departure (AoD) and angle of arrival (AoA) of the l-path, respectively, and (.) is the dot product operator.

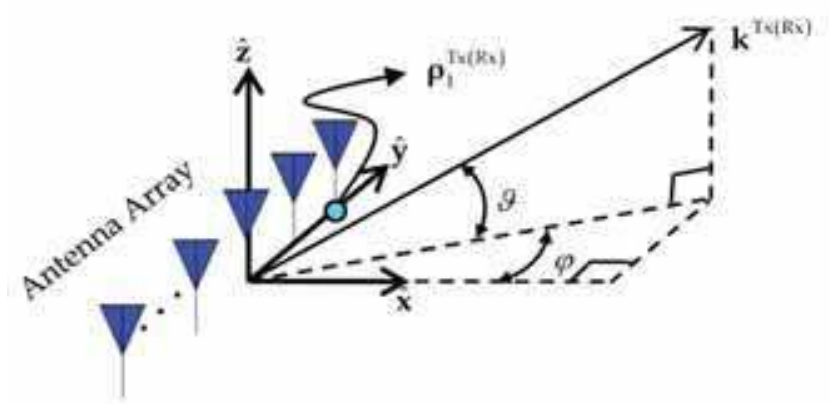

Fig. 2. Representation of wavefronts as vectors in the three-dimensional space.

Each vector $\mathbf{k}_{l}^{\mathrm{Tx}}$ and $\mathbf{k}_{l}^{\mathrm{Rx}}$ can be expressed with the aid of Fig. 2 in spherical coordinates as: $[\cos (\varphi) \cos (\vartheta) \hat{\mathbf{x}}, \sin (\varphi) \cos (\vartheta) \hat{\mathbf{y}}, \sin (\vartheta) \hat{\mathbf{z}}]$. Where angle $\varphi$ is the azimuth angle, defined positive when going counter clock-wise; $\vartheta$ corresponds to the elevation angle. It has also been illustrated in the figure an antenna array located in the direction of $\hat{\mathbf{y}}$ for future 
exemplifications. In the figure, it has been defined the reference point as the position of the first element of the antenna array.

It is worthwhile to mention that equation (3) has been considered that the change of phase depends only on the carrier frequency, when this parameter should be a phase function that depends on the signal (in passband) frequency. However, this approximation is justified for the large ratio between carrier frequency to signal bandwidth (Durgin, 2003).

Transmitting a delta function in order to measure the passband impulse response, and measuring the signal at the receiver point with a wideband quadrature receiver, the received signal is the well known complex baseband impulse response $h($.$) (multiplying the$ quadrature component by the imaginary number $j$ ); this response is dependent of the exact positions of the transceivers, and then it can be defined as:

$$
y(t)=h\left(t, \tau, \mathbf{\rho}_{1}^{\mathrm{Tx}}, \boldsymbol{\rho}_{1}^{\mathrm{Rx}}\right)=\sum_{l=1}^{L_{p}} A_{l} \delta\left(\tau-\tau_{l}\right) e^{-j v_{l} t-j \theta_{l}^{\mathrm{c}}} e^{-j k_{0} \mathbf{k}_{l}^{\mathrm{T} x} \cdot \mathbf{\rho}_{1}^{\mathrm{Tx}}} e^{-j k_{0} \mathbf{k}_{l}^{\mathrm{Rx}} \cdot \mathbf{\rho}_{1}^{\mathrm{Rx}}} f_{l}^{\mathrm{Tx}}(\varphi, \vartheta) f_{l}^{\mathrm{Rx}}(\varphi, \vartheta) .
$$

Where instead of $2 \pi / \lambda$ it has been utilized the wavenumber $k_{0}$. It has been also used the concepts of time-varying filters from (Bello, 1963), therefore, the time domain can be separated into the time and time delay variables to express $\delta\left(t-\tau_{l}\right)$ as $\delta\left(\tau-\tau_{l}\right)$.

Expressions $f_{l}^{\mathrm{Rx}}(\varphi, \vartheta)$ and $f_{l}^{\mathrm{Tx}}(\varphi, \vartheta)$ are, respectively, the response of the measuring system (or underlying antenna array) to the impinging wave, and the transmitter induced distortion to the transmitted signal in the AoD of the $l$-path. Both expressions correspond to the topology construction of the antenna system (Weichselberger, 2003). Hereafter it will be considered that those terms are both equal to unity and are not subsequently considered in the rest of the analysis for ease of explanation.

Equation (4) can be generalized for measuring the signal in a continuous three-dimensional space with the variable $\mathbf{r}^{\mathrm{Rx}}$, from sending signals in every point in the space $\mathbf{r}^{\mathrm{Tx}}$; in this case, the complex baseband channel is:

$$
h\left(t, \tau, \mathbf{r}^{\mathrm{Tx}}, \mathbf{r}^{\mathrm{Rx}}\right)=\sum_{l=1}^{L_{p}} A_{l} \delta\left(\tau-\tau_{l}\right) e^{-j v_{l} t-j \theta_{l}^{\mathrm{c}}} e^{-j k_{0} \mathbf{k}_{l}^{\mathrm{Tx} \times} \cdot \mathbf{r}^{\mathrm{Tx}}} e^{-j k_{0} \mathbf{k}_{l}^{\mathrm{Rx}} \cdot \mathbf{r}^{\mathrm{Rx}}} .
$$

This equation is the generalized form of a complex baseband space-time channel. It is not commonly encountered in literature, as the actual applications are mainly interested in the finite set of points $\left\{\mathbf{r}_{n}^{\mathrm{Tx}}\right\}$ and $\left\{\mathbf{r}_{\mathrm{m}}^{\mathrm{Rx}}\right\}$, where the $\mathrm{n}=1,2, \ldots, \mathrm{N}$ transmitters and the $\mathrm{m}=1,2, \ldots, \mathrm{M}$ receivers are located, for the case of MIMO systems with $\mathrm{M} \times \mathrm{N}$ transceivers. Using (5) for a MIMO system, the previous expression becomes:

$$
h\left(t, \tau, \mathbf{r}_{n}^{\mathrm{Tx}}, \mathbf{r}_{m}^{\mathrm{Rx}}\right)=\sum_{l=1}^{L_{p}} A_{l} \delta\left(\tau-\tau_{l}\right) e^{-j v_{l} t-j \theta_{l}^{c}} e^{-j k_{0} \mathbf{k}_{l}^{\mathrm{Tx}} \cdot \mathbf{r}_{n}^{\mathrm{Tx}}} e^{-j k_{0} \mathbf{k}_{l}^{\mathrm{Rx}} \cdot \mathbf{r}_{m}^{\mathrm{Rx}}}
$$

Arranging the last expression for each of the $\mathrm{M} \times \mathrm{N}$ values in a matrix $\mathbf{H}(t, \tau)$ of $\mathrm{M}$ rows by $\mathrm{N}$ columns entries of two variables each, we have the MIMO channel model useful for system simulations:

$$
\{\mathbf{H}(t, \tau)\}_{m, n}=h\left(t, \tau, \mathbf{r}_{n}^{\mathrm{Tx}}, \mathbf{r}_{m}^{\mathrm{Rx}}\right) ; \quad m=1,2, \ldots, \mathrm{M} ; \quad n=1,2, \ldots, \mathrm{N} .
$$

Equation (7) shows that general MIMO case can be seen as a set of SISO channels for each pair of transmitter and receiver antennas. Therefore a MIMO simulator needs to implement a set of M $\times \mathrm{N}$ SISO channels in a structure as the one shown in Fig. 3, where $h_{m, n}(t, \tau)$ represents the SISO channel between antenna $n$ at Tx and antenna $m$ at $\mathrm{Rx}$, while $x_{1}, \ldots, x_{\mathrm{N}}$ and 
$y_{1, \ldots, y_{\mathrm{M}}}$, represent the respective input and output signals. All signals from the Tx antennas arriving at one $\mathrm{Rx}$ antenna must be added to generate the corresponding Rx output.

Fig. 3. Structure of a MIMO simulator.

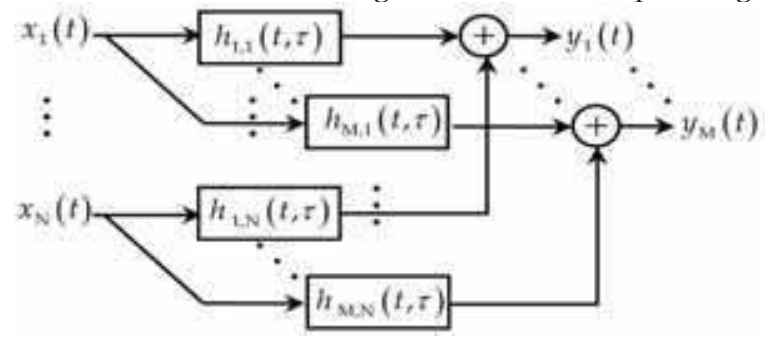

Equation (6) can be simplified to gain insight, considering only linear arrays, located as shown in the Fig. 2 along $\hat{\mathbf{y}}$ direction. With this approach the channel impulse response of the system is measured in the positions $r^{\mathrm{Rx}}$ for signals transmitted along positions $r^{\mathrm{Tx}}$ :

$$
h\left(t, \tau, r^{\mathrm{Tx}}, r^{\mathrm{Rx}}\right)=\sum_{l=1}^{L_{p}} A_{l} \delta\left(\tau-\tau_{l}\right) e^{-j v_{l} t-j \theta_{l}^{c}} e^{-j k_{0} \sin \left(\varphi_{l}\right) r^{\mathrm{Tx}}} e^{-j k_{0} \sin \left(\varphi_{l}\right) r^{\mathrm{Rx}}} .
$$

Or in its general form as:

$$
h\left(t, \tau, r^{\mathrm{Tx}}, r^{\mathrm{Rx}}\right)=\sum_{l=1}^{L_{p}} A_{l} \delta\left(\tau-\tau_{l}\right) e^{-j v_{l} t-j \theta_{l}^{c}} e^{-j k_{l}^{\mathrm{Tx}} r^{\mathrm{Tx}}} e^{-j k_{l}^{\mathrm{Rx}} r^{\mathrm{Rx}}} .
$$

\subsection{Simplified channel description by use of angle domains}

Taking the Fourier transform of (9) with respect to $r^{\mathrm{Tx}}$ and $r^{\mathrm{Rx}}$, we reach the spatial channel expressed in the wavenumber domain, as is explained in detail in (Durgin, 2003):

$$
h\left(t, \tau, k^{\mathrm{Tx}}, k^{\mathrm{Rx}}\right)=\sum_{l=1}^{L_{p}} A_{l} \delta\left(\tau-\tau_{l}\right) \delta\left(k^{\mathrm{Tx}}-k_{l}^{\mathrm{Tx}}\right) \delta\left(k^{\mathrm{Rx}}-k_{l}^{\mathrm{Rx}}\right) e^{-j \nu_{l} t-j \theta_{l}^{\mathrm{c}}} .
$$

In the last expression, it could be considered that all shifts in the wavenumbers should be positive, however, eq. (10) is utilized in accordance with expressions commonly found in literature. This can be used without lose of generality, as the final expression does not depend on this sign. Instead of using an expression that depends on the wavenumber, the most common expressions found are channel impulse responses (CIR) given in terms of the AoD and the AoA. By supposing that the entire propagation scenario lies in a plane with only the azimuth angle taken into consideration (a 2-dimensional propagation environment) we arrive to the following form of the angular dependant CIR,

$$
h\left(t, \tau, \varphi^{\mathrm{Tx}}, \varphi^{\mathrm{Rx}}\right)=\sum_{l=1}^{L_{p}} A_{l} \delta\left(\tau-\tau_{l}\right) \delta\left(\varphi^{\mathrm{Tx}}-\varphi_{l}^{\mathrm{Tx}}\right) \delta\left(\varphi^{\mathrm{Rx}}-\varphi_{l}^{\mathrm{Rx}}\right) e^{-j \nu_{l} t-j \theta_{l}^{c}} ;
$$

where $\varphi^{\mathrm{Tx}}$ and $\varphi^{\mathrm{Rx}}$ are the azimuth angle variables as shown in Fig. 2, while $\varphi_{l}^{\mathrm{Tx}}$ and $\varphi_{l}^{\mathrm{Rx}}$ represents the azimuth AoD and AoA of the $l$-path, respectively. Equation (11) is rather simple, but can be made even simpler by taking its Fourier transform with respect to the $t$ variable, to construct what have been called the double-direction channel model by (Steinbauer et al., 2001): 


$$
h\left(v, \tau, \varphi^{\mathrm{Tx}}, \varphi^{\mathrm{Rx}}\right)=\sum_{l=1}^{L_{p}} A_{l} e^{-j \theta_{l}^{c}} \delta\left(\tau-\tau_{l}\right) \delta\left(\varphi^{\mathrm{Tx}}-\varphi_{l}^{\mathrm{Tx}}\right) \delta\left(\varphi^{\mathrm{Rx}}-\varphi_{l}^{\mathrm{Rx}}\right) \delta\left(v-v_{l}\right)
$$

This expression is really easy to interpret: the radio channel is composed of paths that starts from $\varphi_{l}^{\mathrm{Tx}}$ degrees away the reference angle at the normal of the array, where during its traveling from $\mathrm{Tx}$ to $\mathrm{Rx}$, suffer a delay $\tau_{l}$ with respect to the first incoming wave, and suffers a frequency shift $v_{l}$ from the carrier frequency, and arrives at Rx forming an angle $\varphi_{l}^{\mathrm{Rx}}$ with the normal of the array at the receiver side.

It is possible to pass from (12) to the MIMO channel defined in equation (7), via the transformation from the angle domains to the wavenumber domains and then to the space domain. This approach will be clarified in the subsection 2.4. In this paragraph a direct approach will be considered.

For linear arrays the variables $r^{\mathrm{Tx}}$ and $r^{\mathrm{Rx}}$ can be sampled in the values that define the location of the antennas. In this case, it can be considered the phase difference corresponding to the AoD and AoA of each path in the array, in a vector so-called the Array Manifold Vector (AMV) defined in (VanTrees, 2002). Using the AMV at each link end, the equation that connects realization in the angle domains with the MIMO channel is:

$$
\mathbf{H}(t, \tau)=\sum_{l=1}^{L_{p}} A_{l} \delta\left(\tau-\tau_{l}\right) e^{-j v_{l} t-j \theta_{l}^{\epsilon}} V^{\mathrm{Tx}}\left(\varphi_{l}^{\mathrm{Tx}}\right) V^{\mathrm{Rx}}\left(\varphi_{l}^{\mathrm{Rx}}\right)^{T} ;
$$

where $V^{\mathrm{Tx}(\mathrm{Rx})}$ denotes the AMV at the transmitter Tx $(\mathrm{Rx})$ side, respectively, and $(.)^{T}$ denotes the transposition operator. In the case of a linear antenna array and with the aid of (8), it becomes clear that the AMV takes the following form,

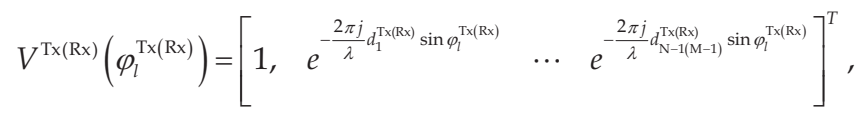

where $d_{1}^{\mathrm{Tx}(\mathrm{Rx})}, \ldots, d_{\mathrm{N}-1(\mathrm{M}-1)}^{\mathrm{Tx}(\mathrm{Rx})}$ denote the position of the antenna elements at the array at Tx and $\mathrm{Rx}$, respectively. Each of these distances has been measured taking the first antenna of the array as the reference point.

An illustration of this approach will be presented to clarify the concepts: consider a $5 \times 3$ ( $N=3$ antennas at $\mathrm{Tx}$ side and $\mathrm{M}=5$ antennas at $\mathrm{Rx}$ side) MIMO system immersed in a propagation scenario which only has $L_{p}=3$ paths, no one with Doppler shift, and its remaining parameters displayed in Table 1, where delays are expressed in $\mu \mathrm{s}$, angles in degrees, and weights in volts. The Antennas are configured in a linear array spaced half wavelength.

\begin{tabular}{ccccc}
\hline \hline l-path & Delay $\tau_{l}$ & Weight $A_{l}$ & AoD $\varphi_{l}^{\mathrm{Tx}}$ & AoA $\varphi_{l}^{\mathrm{Rx}}$ \\
\hline 1 & 0 & $.5+.3 j$ & -10 & -20 \\
2 & 3 & $1+0 j$ & 5 & 10 \\
3 & 6 & $.8-.5 j$ & 20 & 50 \\
\hline
\end{tabular}

Table 1. Parameters of an example to construct a MIMO channel realization 
Using (13), the MIMO channel realization for all the time considered in a block of data consists of three matrices (assuming infinite bandwidth), each one for each of the delays considered. The result for the complex baseband channel for the delay at the second path is presented in the following table. Additionally, the channel realization for the SISO link established in $\mathbf{H}(\tau)_{3,2}$ is, considering infinite channel bandwidth and sampling each microsecond: [-0.5806 - 0.0543j, 0, 0, $0.2045-0.9789 j, 0,0,0.9309-0.1532 j]$ for all $t$.

\begin{tabular}{|c|c|c|c|c|c|c|c|}
\hline $\begin{array}{c}T x \backslash R x \\
\text { Antenna }\end{array}$ & 1 & & 2 & 3 & 4 & 5 & \\
\hline 1 & 1.0000 & & $\begin{array}{l}0.8549 \\
0.5189 j\end{array}$ & $\begin{array}{l}0.4615 \\
0.8871 j\end{array}$ & $\begin{array}{l}-0.0658- \\
0.9978 j\end{array}$ & $\begin{array}{l}-0.5740 \\
0.8189 j\end{array}$ & - \\
\hline 2 & $\begin{array}{l}0.9627 \\
0.2704 j\end{array}$ & - & $\begin{array}{l}0.6827 \\
0.7307 j\end{array}$ & $\begin{array}{l}0.2045 \\
0.9789 j\end{array}$ & $\begin{array}{l}-0.3331- \\
0.9429 j\end{array}$ & $\begin{array}{l}-0.7740 \\
0.6332 j\end{array}$ & - \\
\hline 3 & $\begin{array}{l}0.8538 \\
0.5207 j\end{array}$ & - & $\begin{array}{l}0.4597 \\
0.8881 j\end{array}$ & $\begin{array}{l}-0.0678 \\
0.9977 j\end{array}$ & $\begin{array}{l}-0.5757- \\
0.8177 j\end{array}$ & $\begin{array}{l}-0.9164 \\
0.4003 j\end{array}$ & - \\
\hline
\end{tabular}

Table 2. Parameters of the channel realization $\mathbf{H}(t, \tau)^{T}$ for $\tau=3 \mu$ s an any $t$

In Table 2, $\mathbf{H}($.$) of size \mathrm{M} \times \mathrm{N}$ have been presented transposed. It is important to stress here, that with the former discussion, given channel realizations in the angle domains, channel realization at the space domain can be carried out.

\subsection{Statistical description of the MIMO Radio Channel}

As $\theta_{l}^{c}=\omega_{c} \tau_{l}$ is the phase of the received carrier wave, and each delay is not commensurate with the carrier wave period, this quantity should be regarded as random and uniform between $[-\pi, \pi)$, as discussed in detail in (Kennedy, 1969). It can be considered that several paths are clustering into a set of $L$ principal paths, and then, the summation in (10) can be represented for the channel expressed in the wavenumber domains and the angular domains as (15) and (16), respectively:

$$
\begin{aligned}
& h\left(t, \tau, k^{\mathrm{Tx}}, k^{\mathrm{Rx}}\right)=\sum_{l=1}^{L} \alpha_{l}^{k} \delta\left(\tau-\tau_{l}\right) \delta\left(k^{\mathrm{Tx}}-k_{l}^{\mathrm{Tx}}\right) \delta\left(k^{\mathrm{Rx}}-k_{l}^{\mathrm{Rx}}\right) e^{-j v_{l} t}, \\
& h\left(t, \tau, \varphi^{\mathrm{Tx}}, \varphi^{\mathrm{Rx}}\right)=\sum_{l=1}^{L} \alpha_{l} \delta\left(\tau-\tau_{l}\right) \delta\left(\varphi^{\mathrm{Tx}}-\varphi_{l}^{\mathrm{Tx}}\right) \delta\left(\varphi^{\mathrm{Rx}}-\varphi_{l}^{\mathrm{Rx}}\right) e^{-j v_{l} t} .
\end{aligned}
$$

Where $\alpha_{l}, \alpha_{l}^{k}$ are complex Gaussian random variables with zero mean, and was obtained from the sum of several complex sinusoids within the same cluster (paths that departs mainly from the same angle, arrive at the same angle, suffers almost the same delay and Doppler shift, but traveled by separate ways). Those sums involves the sum of complex exponential with random phase, which rapidly tends to a Gaussian distribution, provided in practice that we deal with more than five waves (Pätzold, 2002). It is worthwhile to note that the main difference within $\alpha_{l}$ and $\alpha_{l}^{k}$ are their variances.

Transforming equation (15) in the sense of Fourier for the $t$ variable, we arrive to the stochastic model in the wavenumber, delay and Doppler domains: 


$$
h\left(v, \tau, k^{\mathrm{Tx}}, k^{\mathrm{Rx}}\right)=\sum_{l=1}^{L} \alpha_{l}^{k} \delta\left(\tau-\tau_{l}\right) \delta\left(v-v_{l}\right) \delta\left(k^{\mathrm{Tx}}-k_{l}^{\mathrm{Tx}}\right) \delta\left(k^{\mathrm{Rx}}-k_{l}^{\mathrm{Rx}}\right) .
$$

And applying the same transformation in (16) we arrive to the stochastic description of the bi-directional channel model:

$$
h\left(v, \tau, \varphi^{\mathrm{Tx}}, \varphi^{\mathrm{Rx}}\right)=\sum_{l=1}^{L} \alpha_{l} \delta\left(\tau-\tau_{l}\right) \delta\left(v-v_{l}\right) \delta\left(\varphi^{\mathrm{Tx}}-\varphi_{l}^{\mathrm{Tx}}\right) \delta\left(\varphi^{\mathrm{Rx}}-\varphi_{l}^{\mathrm{Rx}}\right) .
$$

For Gaussian processes, second order statistics completely describes the process. We hereafter consider only the double-directional channel model, but the same results directly apply to the model expressed by equation (17), that is dependent on wavenumber domains. The connection between correlation of the former expressions, i.e. the one that depends in angles and the one that depends on wavenumbers, will be explained in the following section.

Taking the autocorrelation function of last equation, we arrive to a multidimensional function than can be called the Multidimensional Power Density Spectrum of the doubledirectional (bi-directional) channel (MPDSB) $S_{B h}($.$) :$

$$
S_{B h}\left(v, v^{\prime} ; \tau, \tau^{\prime} ; \varphi^{\mathrm{Tx}}, \varphi^{\mathrm{Tx}{ }^{\prime}} ; \varphi^{\mathrm{Rx}}, \varphi^{\mathrm{Rx}{ }^{\prime}}\right)=E\left\{h\left(v, \tau, \varphi^{\mathrm{Tx}}, \varphi^{\mathrm{Rx}}\right) h^{*}\left(v^{\prime}, \tau^{\prime}, \varphi^{\mathrm{Tx}{ }^{\prime}}, \varphi^{\mathrm{Rx}}\right)\right\} ;
$$

where $E\{$.$\} is the expected value operator and (..) is the complex conjugate operator. The$ apostrophes are utilized to define auxiliary variables for each domain. This autocorrelation function is too complex to be treated, and its information is hard to count with. It is justified in (Durgin, 2003) that the channel can be regarded for several scenarios as uncorrelated in all domains, the last expression can then be expressed in terms of fewer variables, here called as the Multidimensional Scattering Function of the Bidirectional Channel (MSFB) $S_{B}($.$) in the$ following way:

$$
S_{B h}\left(v, v^{\prime} ; \tau, \tau^{\prime} ; \varphi^{\mathrm{Tx}}, \varphi^{\mathrm{Tx}{ }^{\prime}} ; \varphi^{\mathrm{Rx}}, \varphi^{\mathrm{Rx} \prime^{\prime}}\right)=S_{B}\left(\nu, \tau, \varphi^{\mathrm{Tx}} \varphi^{\mathrm{Rx}}\right) \delta\left(\tau-\tau^{\prime}\right) \delta\left(\nu-v^{\prime}\right) \delta\left(\varphi^{\mathrm{Tx}}-\varphi^{\mathrm{Tx} \prime}\right) \delta\left(\varphi^{\mathrm{Rx}}-\varphi^{\mathrm{Rx}{ }^{\prime}}\right) .
$$

Which considering only the values where the last function is different from zero (the diagonals of each correlation in the domains of each variable and its auxiliary variable) collapses in the multipath description to:

$$
S_{B}\left(v, \tau, \varphi^{\mathrm{Tx}}, \varphi^{\mathrm{Rx}}\right)=\sum_{l=1}^{L} \sigma_{l} \delta\left(\tau-\tau_{l}\right) \delta\left(v-v_{l}\right) \delta\left(\varphi^{\mathrm{Tx}}-\varphi_{l}^{\mathrm{Tx}}\right) \delta\left(\varphi^{\mathrm{Rx}}-\varphi_{l}^{\mathrm{Rx}}\right) .
$$

In the last equation, $\sigma_{l}=E\left\{\alpha_{l} \alpha_{l}^{*}\right\}$ is the variance of the $l$-path. The taken simplification had assumed that all $l$-paths are uncorrelated between them, which in turn implies that in their dual domains are all stationary (Bello, 1963). These MSFB though is a simplification of the MPDSB is still complex to be measured and characterized; so, it is not strange to encounter partial description of it.

First of all, it will be taken into account only the temporal variables in the MSFB, which can be achieved via the integration of (21) for all AoA and AoD. This function can be also defined by taking the average intensity profile in the delay and Doppler variables for each SISO link in the MIMO channel. Using this delay and Doppler statistic information, it has been defined the well known Scattering Function (SF). This function provides a distribution 
of the signal-strength averages, for all incoming paths, in terms of delays and frequency shifts. An example of it is illustrated in Fig. (4a). The plotted SF has been taken from the COST-BU channel standard (COST 207, 1989) and the path energies have been normalized to the maximum energy. The delay axis $(\tau)$ is graded in $\mu \mathrm{s}$, while the Doppler shift axis $(v)$ is in Hz. In the figure, the SF has been further simplified into a set of identifiable paths via paths integration for paths in the neighborhood of the chosen delay for the main path. The Doppler Spectrum of each path is shown in Fig. (4b), while its average power is plotted in Fig. (4c).

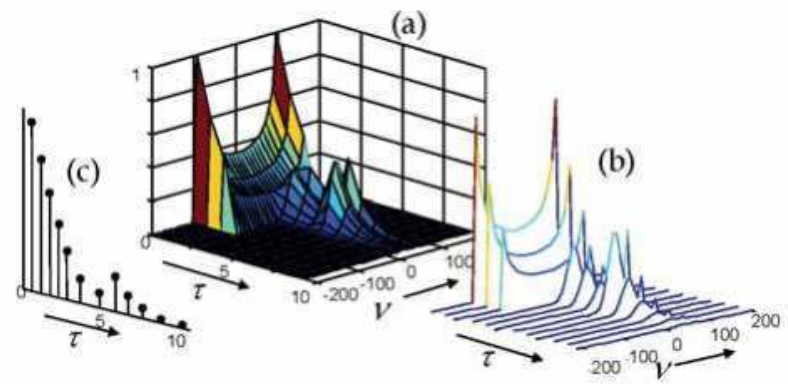

Fig. 4. The SF and its simplification by integration into finite set of paths.

Other simplification of the MSFB can provide only the information of the angular variables, which can be obtained through integration of the MSFB for all delays and Doppler shifts, as in:

$$
\iint_{\tau} S_{B}\left(v, \tau, \varphi^{\mathrm{Tx}}, \varphi^{\mathrm{Rx}}\right) d v d \tau=S\left(\varphi^{\mathrm{Tx}}, \varphi^{\mathrm{Rx}}\right) ;
$$

the resulting functions can be called the Cross Power Azimuth Spectrum (xPAS). This function specifies what is the PAS at Rx for the transmitted signal at each angle at Tx. An example of a PAS is given in Fig. (5a). Integrating the MSFB for all angles at the transmitter side and all Doppler Shifts. The resulting function is called the Azimuth-Delay Power Spectrum (ADPS) function, and it provides the average profile for paths at each delay and azimuth AoA. See for instance Fig. (5b). On averaging the ADPS function for all delays, we obtain the PAS, while on averaging all the AoAs we obtain the Power Delay Profile (PDP). These two profiles are shown in Figs. (5a \& c), respectively.

(b)

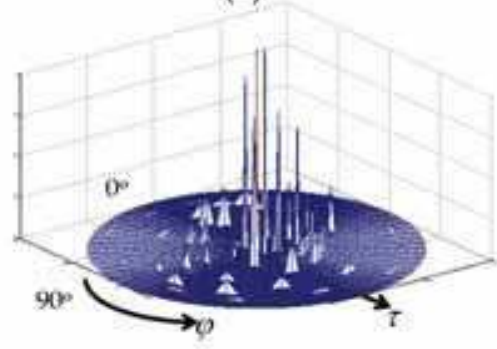

(a)

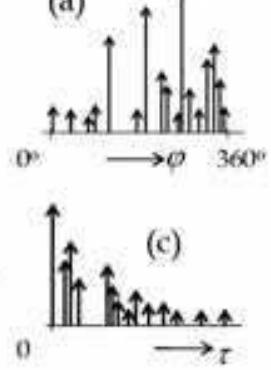

Fig. 5. Multipath intensity profile for angle and delay variables. 
If in the explanation, it would have been utilized the channel function that depends on the wavenumbers, we had arrived to the Multidimensional Power Density Spectrum of the Spatial Channel (MPDSS). Using the uncorrelated assumption, its simplification in terms of the Multidimensional Scattering Function of the Spatial Channel (MSFS) $S_{S}($.$) would be$ reached. From this expression, it is possible to integrate the MSFS for delay and Doppler variables, to obtain the cross Wavenumber Spectrum (xWNS), in the following way:

$$
\iint_{v} S_{S}\left(v, \tau, k^{\mathrm{Tx}}, k^{\mathrm{Rx}}\right) d v d \tau=S\left(k^{\mathrm{Tx}}, k^{\mathrm{Rx}}\right) .
$$

\subsection{The Gans Mapping tool to pass from PAS functions to WNS}

It has been already discussed how to pass from channel realization in the angular domain, to channel realizations in the spatial domain: through the use of the AMV. If the channel realizations are performed in the wavenumber or wavevector domains, the connection to the spatial domain is via a Fourier transform. In this subsection, it will be discussed how to connect PAS and WNS with autocorrelations in the space domain.

The Gans Mapping tool (GM), relate the expressions between angle and spatial domains. It was proposed in (Gans, 1972), but reintroduced in the modeling literature by Durgin; see (Durgin, 2003) and references there in. As it has been discussed, the PAS is one of the most important concepts in spatial channel modeling since it provides a simpler and more intuitive way to characterize the spatial channel than in terms of WNS. In its detriment, we can mention that the PAS is not the natural domain for relating spectral properties to spatial selectivity in a channel. Therefore, the PAS must be converted to a wavevector spectrum (or in this case WNS) whenever spatial correlations, spectral spreads or duality results are calculated. This means, having some PAS, the WNS can be obtained through GM and then the Spatial Correlation Function (SCF) is calculated via the Fourier transform of the WNS. It should be noticed that the information of spatial channels is not commonly provided in standards or sounding campaigns; instead of this, PAS functions are considered in the sounding measurement campaigns.

Given a (one dimensional) PAS $p(\varphi)$, it is possible to calculate the corresponding WNS by:

$$
S(k)=2 \pi \frac{p\left(\varphi_{R G}+\cos ^{-1}\left(k / k_{0}\right)\right)+p\left(\varphi_{R G}-\cos ^{-1}\left(k / k_{0}\right)\right)}{\sqrt{k_{0}-k^{2}}} .
$$

where $\varphi_{R G}$ is the azimuthal direction of movement, $S(k)$ is the WNS, and $|k| \leq k_{0}$. These parameters are graphically shown with the aid of Fig. 6. 


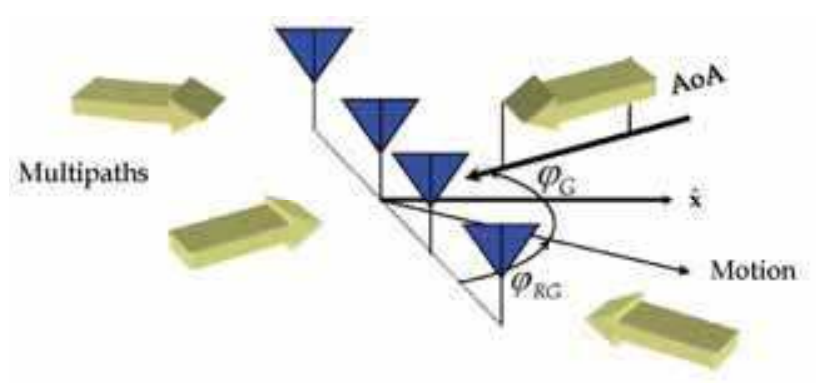

Fig. 6. The multipath components arriving at the antenna array.

The mapping given at equation (24) has a straight-forward physical interpretation from the propagation shown at Fig. 6. A multipath of plane wave arrives from the horizon at an angle $\varphi_{G}$ and the direction of azimuthally motion that we wish to map is $\varphi_{R G}$. The phase progression of this multipath wave is the free space wavenumber $k_{0}=2 \pi / \lambda$. However, to a receiver moving along the $\varphi_{R G}$ direction, the actual wavenumber appears to be foreshortened by the factor $k_{0} \cos \left(\varphi_{G}-\varphi_{R G}\right)$ (Durgin, 2003). This mapping was utilized to analyze Doppler Spectra of a single Antenna considering some PAS and receiver movement. But it can be also utilized to analyze the WNS of an antenna array considering that the receiver array is located in the direction of the movement (or vice versa). Comparing this figure with Fig. 2, it becomes clear that $\varphi_{\mathrm{G}}$ in Fig. 6 is $\varphi+90^{\circ}$ in Fig. 2.

Fig. 7 shows an example of the GM application. The particular form of the PAS is shown in Fig. (7a). The associated WNS in Fig. (7b) is obtained directly from the use of the GM over the PAS. The real and imaginary parts of the SCF (Fig. (7c) and Fig. (7d), respectively) are finally obtained through a Fourier transform on the WNS; a comparison to the result given in terms of closed form expression at (Salz \& Winters, 1994) is also included in Fig. (7c) and (7d). In (Alcocer-Ochoa et al., 2008) the same SCF was presented with the use of the AMV.

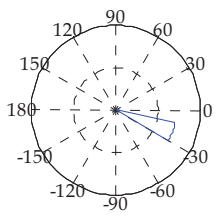

(a) PAS

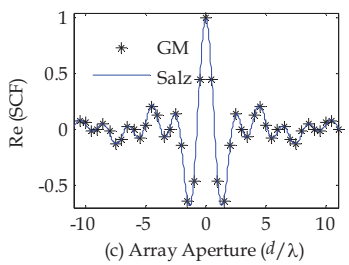

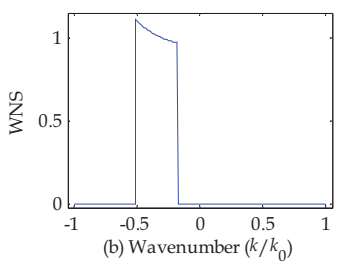

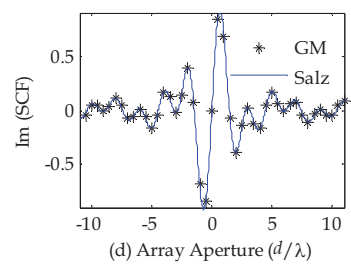

Fig. 7. The WNS and its SCF for a limited uniform distributed PAS. 
Another approach to calculate the SCF is by departing from the PAS; it is performed by using the AMV. Consider that channel realizations only depends on the angular variables for ease of explanation:

$$
h\left(\varphi^{\mathrm{Tx}}, \varphi^{\mathrm{Rx}}\right)=\sum_{l=1}^{L} \alpha_{l} \delta\left(\varphi^{\mathrm{Tx}}-\varphi_{l}^{\mathrm{Tx}}\right) \delta\left(\varphi^{\mathrm{Rx}}-\varphi_{l}^{\mathrm{Rx}}\right) .
$$

The spatial values can be obtained with the use of the AMV. Consider the case of sampled points in the space for the construction of a MIMO channel, then:

$$
\mathbf{H}=\sum_{l=1}^{L} \alpha_{l} V^{\mathrm{Tx}}\left(\varphi^{\mathrm{Tx}}{ }_{l}\right) V^{\mathrm{Rx}}\left(\varphi_{l}^{\mathrm{Rx}}\right)^{T} .
$$

The spatial autocorrelation tensor of the MIMO function $R_{H}$ can be obtained through:

$$
R_{H}=E\left\{H \times H^{H}\right\}=E\left\{\sum_{l=1}^{L} \alpha_{l} V^{\mathrm{Tx}}\left(\varphi^{\mathrm{Tx}}{ }_{l}\right) V^{\mathrm{Rx}}\left(\varphi^{\mathrm{Rx}}{ }_{l}\right)^{T} \times\left(\sum_{l^{\prime}=1}^{L} \alpha_{l^{\prime}} V^{\mathrm{Tx}}\left(\varphi_{l^{\prime}}^{\mathrm{Tx}{ }^{\prime}}\right) V^{\mathrm{Rx}}\left(\varphi^{\mathrm{Rx}{ }^{\prime}{ }^{\prime}}\right)^{T}\right)^{H}\right\} .
$$

Where $(.)^{H}$ denotes conjugate transpose (Hermitian), $(\times)$ stands for the outer product, and the variables with the apostrophe are auxiliary variables. Invoking the uncorrelated assumption, we finally arrive to:

$$
R_{H}=\sum_{l=1}^{L} \sigma_{l} V^{T x}\left(\varphi^{T x}{ }_{l}\right) V^{T x}\left(\varphi^{T x}{ }_{l}\right)^{H} \times V^{R x}\left(\varphi^{R x}{ }_{l}\right) V^{R x}\left(\varphi^{R x}{ }_{l}\right)^{H} .
$$

In the last equation, we have used the fact that $V^{\mathrm{Tx}}\left(\varphi^{\mathrm{Tx}}{ }_{l}\right) V^{\mathrm{Rx}}\left(\varphi^{\mathrm{Rx}}{ }_{l}\right)^{T}$ are also external products. It is worthwhile to mention that the results of the sums on $V^{\mathrm{Tx}}\left(\varphi^{\mathrm{Tx}}{ }_{l}\right) V^{\mathrm{Tx}}\left(\varphi^{\mathrm{Tx}}{ }_{l}\right)^{H}$ become matrices of dimension $\mathrm{N} \times \mathrm{N}$, and then $R_{H}$ is a tensor of $(\mathrm{M} \times \mathrm{N})$ $\times(\mathrm{M} \times \mathrm{N})$ entries.

\subsection{Determination of the channel modeling and simulation problem}

From last expressions, it can now be state the problem that concerns channel modeling and simulation:

Channel Modeling: To conceive propagation models and mathematical expressions that justifies the shapes of the measured statistics in the MSFB. It means people focused on channel modeling area had tried to answer the following questions: Why in an urban environment the time correlation function tends to a Bessel Function? Which scattering model is underlying when the received PAS is Gaussian type?

Simulation: To conceive expressions based on the channel models which lead to mathematical expressions and algorithms suitable for its implementation as SW routines or HW implementations. People related to simulation of channels try to figure out how to produce channel realizations with prescribed statistics for all variables of interest; i.e. angles, time delay, etc.

It is worthwhile to note that in the previous discussion, it has been followed a channel representation consisting of a finite number of paths; this approach cannot be directly utilized when the channel behaves as a continuum in the variable of interest; i.e. when the PAS is a continuous function. Approaches to overcome this problem will be discussed. 
Another subject of simulation is to perform the process in the best (economical) way. In HW implementation, it's also a matter of research to settle what other trade-offs should be taken into account to guarantee the emulators will provide the same results than obtained in SW (which have infinite variable resolution).

In the remaining of this chapter, we will be concerned with only the spatial characteristics of the channel. We will also be restricted to linear arrays of finite dimension.

\section{The channel modeling approaches in literature}

The spatio-temporal models can be generally classified into two groups: deterministic and stochastic (Molisch, 2004).

Within the deterministic models, the CIR is obtained by tracing the reflected, diffracted and scattered rays, with the help of databases that provide information about the size and location of the physical structures in addition to the electromagnetic properties of their materials. Deterministic models have the advantage of providing the ability to generate accurate site specific and easily reproducible information; it is also helpful to propose some environment and to measure whatever it supplies.

Stochastic models, on the other hand, describe characteristics of the radio channel by means of the joint PDF and/or correlation functions. Statistical parameters employed in such models are usually estimated from extensive measurement campaigns or inferred from electromagnetic laws and geometrical assumptions. Stochastic models usually need less information than deterministic ones, and produce more general results, as many repetitions are considered.

There are many works related to this approaches, where classifications of the several approaches can be found, such as (Piechocki et al., 2001) and (Molisch et al., 2006). In what follows, we will discuss only one approach of determinist modeling, the geometrical modeling, and only one approach of stochastic modeling, which is the method of artificial paths. The rationale behind this is that geometrical models are useful to provide information that can be interpreted as MDSF, or ADPS, PAS, PDP, etc. In the other hand, the method of artificial paths provides the underlying approach to construct the best simulators, and they only depend on the commented profiles. With these two approaches, a MIMO simulator can be entirely constructed.

\subsection{Geometrical modeling}

The geometrical model tries to explain or construct functions that reflect the average behavior of the multipath phenomena in some environment, such as a PAS, by establishing a Tx and Rx in a scenario surrounded by scatterers. The spatial pattern of scatters is the principal actor in producing the statistics in the multipath, and thus, its specification and relation to real environments are of great interest.

A scatterer is an omni-directional reradiating element whereby the plane wave, on arrival, is assumed to be reflected directly to the mobile receiver antenna or to another scatterer without the influence from any other (Petrus, 2002).

Most of the existing geometric channel models take into account only the local scattering clusters as in (Ertel \& Reed, 1999), which are always located around the mobile station (MS) with few available models defining the shape and distribution of far (or dominant) clusters. The geometrical channel models are well suited for simulations that require a complete 
model of the wireless channel, due to its ray-tracing nature. However, the shape and size of the scatterers' PDF required to achieve a reliable simulation of the propagation phenomenon is still subject to debate.

If only one discrete scatterer is present (the Single Scattering Model in (Laurila et al., 1998)), a closed form expression can be easily found from the signal at the receiver by using fundamental wave propagation and scattering laws. However, if many objects are present, things become much more complicated, since the interaction between different objects must be accounted for. As an example, consider the scenario showed at Fig. 8; in there, the MS is surrounded by scatterers uniformly distributed. This scenario can be observed when the antenna at the base station at $\mathrm{Tx}$ is high enough, so there are no scatterers surrounding it, like in the cellular environment. In order to simplify the analysis, it will be assumed that the scatterers only change the direction of the impinging waves. The parameters of interest in this scenario are the Delay or Time of Arrival (ToA) and the AoA ( $\varphi_{l}^{\mathrm{Rx}}$, in Fig. 8) Probability Density Functions (PDF). Those PDFs will be calculated from the $\operatorname{AoD}\left(\varphi_{l}^{\mathrm{Tx}}\right.$, in Fig. 8) and the position $r$ of the scatterers. Finally, D in Fig. 8 correspond to distance from Tx to Rx. Note that the joint PDFs of the ToA and AoA can be directly interpreted as the ADPS, and then, from this function a SCF can be obtained.

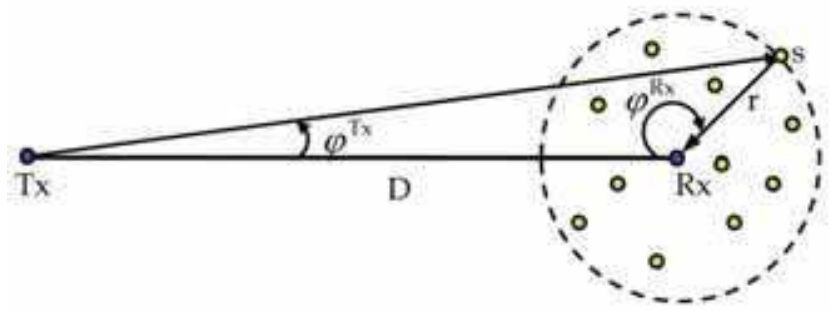

Fig. 8. Uniformly distributed scatterers around the Rx.

In order to obtain the joint ToA and AoA PDFs for this scenario, we consider the comments made in (Laurila et al., 1998) as well as the PDF transformation rules described in (Papoulis \& Pillai, 2002). In such case, the joint Delay and AoA PDF is given by

$$
p\left(c \tau, \varphi^{\mathrm{Rx}}\right)=\frac{1}{\left(R^{\mathrm{Rx}}\right)^{2}} \frac{\left[(c \tau)^{2}+\mathrm{D}^{2}\right] p\left(\varphi^{\mathrm{Tx}}\right)}{(c \tau)-D \cos \left(\varphi^{\mathrm{Rx}}\right)}\left|\mathrm{J}\left(c \tau, \varphi^{\mathrm{Rx}}\right)\right|,
$$

where the Jacobian of the transformation $\mathrm{J}\left(c \tau, \varphi^{\mathrm{Rx}}\right)$ is defined below, and $R^{\mathrm{Rx}}$ denotes the radii where the scatterers lie in.

$$
\mathrm{J}\left(c \tau, \varphi^{\mathrm{Rx}}\right)=\frac{(c \tau)^{4}-3(c \tau)^{2} \mathrm{D}^{2}+2(c \tau) \mathrm{D}\left[\mathrm{D}^{2}-(c \tau)^{2}\right] \cos \left(\varphi^{\mathrm{Rx}}\right)+\mathrm{D}^{2}\left[(c \tau)^{2}+\mathrm{D}^{2}\right] \cos \left(2 \varphi^{\mathrm{Rx}}\right)}{2\left[(c \tau)-\mathrm{D} \cos \left(\varphi^{\mathrm{Rx}}\right)\right]^{2}\left[\mathrm{D}^{2}+(c \tau)^{2}-2(c \tau) \mathrm{D} \cos \left(\varphi^{\mathrm{Rx}}\right)\right]} .
$$

Fig. 9 shows a plot of this joint PDF supposing a uniform AoD distribution, $\mathrm{R}=30 \mathrm{~m}$ is the radio where the scatterers lie, and also $\mathrm{D}=500 \mathrm{~m}$, typical values for picocell scenarios. 


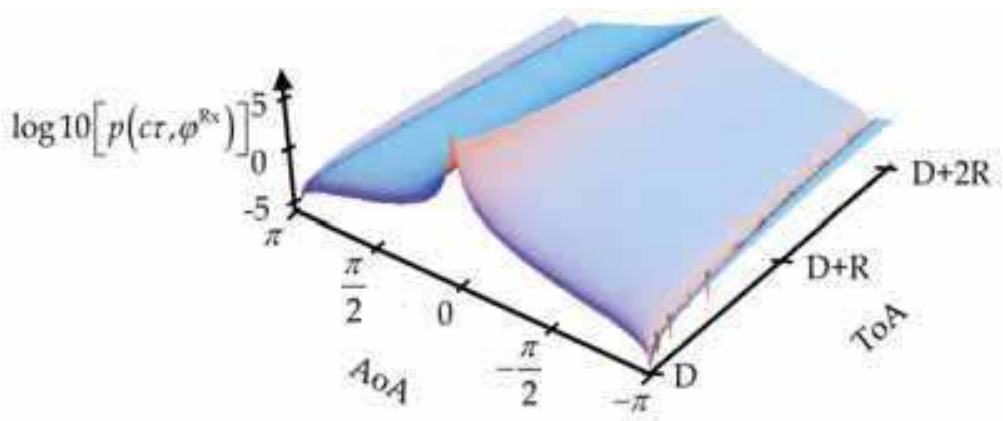

Fig. 9. The joint Delay and AoA for the scenario presented in Fig. 8.

From the previous figure, it seems that the most probable arrivals are those with a relatively low AoA. Observe also that integrating the function for all time delay, a PAS can be conceived. In this case, the PAS closely resemblance to a Gaussian type function, while the PDP can be well approximated by a decaying exponential functions, which is in agreement with common propagation standards, such as COST Typical Urban. Note also that the space and time delay domains are not separable.

Finally, the PDF analysis with the Jacobian method takes into account only those scatterers which are directly illuminated and neglects those which are not. A uniform spatial distribution of scatterers does not lead to a uniform distribution for the AoA. Actually, those distributions of scatterers that lead to this special case of the AoA are difficult to justify physically (Fuhl et al., 1998).

\subsection{Method of artificial paths}

It has been stated through this Chapter, that when the channel is composed of a finite number of paths, its statistics and calculation of the SCF are easy to obtain. However, when the number of physical paths is large, the direct approach cannot be longer used. This could happen, for example if the channel behaves as a continuum instead of identifiable paths. In such environments, we can model the channel as if it were composed by a finite number of paths, with no physical meaning, but reduced in number. In this case, the channel can be expressed as:

$$
h\left(\varphi^{\mathrm{Tx}}, \varphi^{\mathrm{Rx}}\right)=\sum_{c=1}^{C} \beta_{c} \psi_{c}\left(\varphi^{\mathrm{Tx}}, \varphi^{\mathrm{Rx}}\right),
$$

where $\beta_{c}$ are the complex weights of the artificial paths, and are complex Gaussian random variables; $c=1,2 \ldots, C$ is the indexing variable and $C$ denotes the number of terms utilized in the approximation. Functions $\psi_{c}\left(\varphi^{\mathrm{Tx}}, \varphi^{\mathrm{Rx}}\right)$ are the artificial paths. Note that with this model, the paths could not be deltas, it means, they could not point to a specific angle; besides this, there are not guarantee that these complex paths are uncorrelated. The main problem of this approach consists in defining the complex paths and the statistics of its weights in order to be capable to generate channel realizations with the same first and second order statistics that the targeted channel. The approach of these artificial paths was defined long ago in the time-delay domain, see for instance (Fechtel, 1993) and (Parra-Michel et al., 2003); although 
it has been proved to be a good modeling method for the variables of the space channels, as shown in (Kontorovich et al., 2008).

\section{Simulation and Emulation construction of Channel models}

In what follows, we will discuss how simulators can be constructed from the channel models discussed.

\subsection{Statistical Paths generation}

This is a rather simple approach to generate MIMO CIR realizations at the literature (Molisch, 2004). For the spatial domain this approach consist on the generation of ray paths with an Angle of Departure (AoD) and an Angle of Arrival (AoA) by random variable realizations following the suggested statistics at the standards. This statistics can be drawn from information provided by geometrical models, although the converse is also useful: generation of paths with some statistics in the AoD and upon defining some scatterers' distribution, new statistics at $\mathrm{Rx}$ can be devised with the aid of simulations. This approach is complementary to the analytical case illustrated in the geometrical models.

To generate realizations of a random variable with a predefined $\operatorname{PDF} f(\varphi)$, we use the general method described in (Papoulis \& Pillai, 2002), which works as follows: once the PDF is chosen, the Cumulative Distribution Function (CDF) $F(\varphi)$ must be calculated by a simple integration. A plot of a Gaussian CDF is presented in Fig. 10. The method says that once we have a uniformly distributed random variable realization, $u \in[0,1]$, the result from $v=F^{-1}(u)$, will follow the predefined PDF.

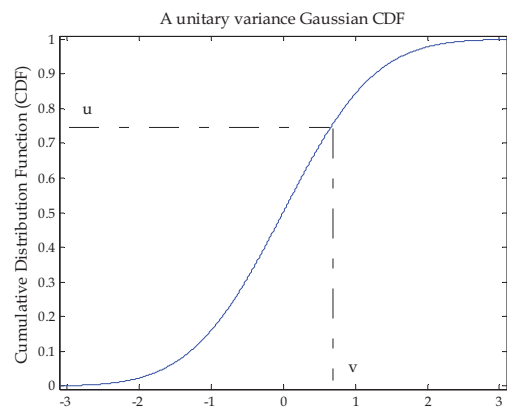

Fig. 10. A CDF calculated from a unitary variance Gaussian PDF.

As an example for the use of the general method, let us consider a 6 path ray tracing scenario which follows a Gaussian distribution. With the use of the Fig. 10, we toss 6 uniformly distributed random variable realizations: [0.9171; 0.7582; 0.8870; 0.0688; 0.1835; 0.7371], then the associated Gaussian distributed random variables are: [1.3802; 0.6989; $1.2068 ;-1.4799 ;-0.8999 ; 0.6329]$. Multiplying this result with the desired variance will yield the desired values. The main advantage of the MIMO CIR realizations method described above, is its intuitive and easy way to generate CIR realizations with the desired statistics. In the literature there is a set of functions proposed for the AoA and ToA modeling, which 
mainly depend on the propagation scenario. The list of the most used forms AoA PDF in Table 3, where $\operatorname{Erf}(\cdot)$ is the Error function and $\mathrm{I}_{0}(\cdot)$ is the $0^{\text {th }}$ order modified Bessel function.

\begin{tabular}{ccc}
\hline \hline Name & Formula & Parameters \\
\hline Uniform & $f(\varphi)=\frac{1}{\varphi_{m x}-\varphi_{m n}}, \quad \varphi_{m n}<\varphi \leq \varphi_{m x}$ & Interval: $\left(\varphi_{m n}, \varphi_{m x}\right]$ \\
Laplacian & $f(\varphi)=\frac{e^{-\frac{1}{\kappa}|\varphi|}}{2 \kappa\left(1-e^{-\frac{\pi}{\kappa}}\right)},-\pi<\varphi \leq \pi$ & Decay Parameter: $\kappa$ \\
Gaussian & $f(\varphi)=\frac{e^{-\frac{1}{2 \sigma^{2} \varphi^{2}}}}{\sqrt{2 \pi \sigma^{2}} E r f\left(\frac{\pi}{\sqrt{2 \pi \sigma^{2}}}\right)}, \quad-\pi<\varphi \leq \pi$ & Variance: $\sigma^{2}$ \\
Von Mises & $f(\varphi)=\frac{e^{-\kappa \cos \varphi}}{2 \pi I_{0}(\kappa)},-\pi<\varphi \leq \pi$ & Parameter: $\kappa$ \\
\hline
\end{tabular}

Table 3. The most commonly used functional forms for the AoA

The 3GPP and 3GPP2 (3GPP, 2003), as an example, propose the use of the uniform distribution (f1) for the pedestrian and the truncated Laplacian distribution (f2) for the vehicular cases. For the urban and suburban macrocell environments, the standards recommend the use of a truncated Gaussian distribution (f3) and uniform distribution for the urban microcell environment. Many standards and recommendations suggest the use of these PDF for its use in several scenarios and operation frequencies. Clearly, the maximum angular spread depends on the particular scenario, and can be estimated from geometrical modeling of the radio propagation channel.

Even thought the von Mises PDF is not proposed at the standards, it has the advantage that approaches to a Gaussian distribution with variance $1 / \kappa$ when $\kappa$ is relatively large, approaches to a Laplacian distribution when $\kappa$ is large; and when $\kappa$ approaches to zero, a von Mises RV tends to be uniform distributed.

\subsection{Paths integration}

Within this approach, we can include those works intended to utilize the model of artificial paths, but using the delta functions for $\psi_{c}\left(\varphi^{\mathrm{Tx}}, \varphi^{\mathrm{Rx}}\right)$. It means, the simulator still tries to reproduce the channel statistics but with an approximation as:

$$
h\left(\varphi^{\mathrm{Tx}}, \varphi^{\mathrm{Rx}}\right)=\sum_{c=1}^{c} \beta_{c} \delta\left(\varphi^{\mathrm{Tx}}-\varphi_{c}^{\mathrm{Tx}}\right) \delta_{c}\left(\varphi^{\mathrm{Rx}}-\varphi_{c}^{\mathrm{Rx}}\right),
$$

where the problem now becomes the selection of weights and angles for the finite set of paths, for a given XPAS. Once given the sets of angles and weights, a channel realization is then composed via:

$$
\mathbf{H}_{p I}=\sum_{c=1}^{c} \beta_{C} V_{c}^{\mathrm{Tx}}\left(\varphi_{c}^{\mathrm{Tx}}\right) V_{c}^{\mathrm{Rx}}\left(\varphi_{c}^{\mathrm{Rx}}\right)^{T}
$$


The general form of the approaches deal with separable functions in the angular domain, which means that XPAS can be represented via the outer product of two one-dimensional functions, in the way of:

$$
S\left(\varphi^{\mathrm{Tx}}, \varphi^{\mathrm{Rx}}\right)=S\left(\varphi^{\mathrm{Tx}}\right) S\left(\varphi^{\mathrm{Rx}}\right),
$$

where each channel with a given PAS is then approximated via a set of reduced number of paths, for example, for the case of Tx it becomes:

$$
h\left(\varphi^{\mathrm{Tx}}\right)=\sum_{c=1}^{C^{T x}} \beta_{c}^{\mathrm{Tx}} \delta\left(\varphi^{\mathrm{Tx}}-\varphi_{c}^{\mathrm{Tx}}\right),
$$

and each weight $\beta_{c}^{\mathrm{Tx}}$ is obtained by an integration of the physical paths, i.e.

$$
\sigma_{c}^{\mathrm{Tx}}=E\left\{\beta_{c}^{\mathrm{Tx}}\left(\beta_{c}^{\mathrm{Tx}}\right)^{*}\right\}=\int_{\varphi_{c}} S\left(\varphi^{\mathrm{Tx}}\right) .
$$

Where the last equation means that the integration of paths assigns to the c-path some of the paths of the PAS. For example, we can select $C_{c}^{\mathrm{Tx}}$, and the variance of each path equal to $1 / C_{c}^{\mathrm{Tx}}$, and turn to find the set of values in the angle domain that delimitate the defined area. Finally, select the angle for the c-path that is at the middle of the integrated area. This method is known as Method of Equal Areas (MEA), and is discussed in detail in (Pätzold, 2002).

If we restrict the integration in such a way that the paths integrated for one of the artificial paths do not belong to any of the other artificial paths for all $C_{c}^{\mathrm{Tx}}$, in this way, weights $\beta_{c}^{\mathrm{Tx}}$ are all uncorrelated and for the model we have presumed, Gaussian as well. Using this separable assumption, in the approximation of (32), each of the weights $\beta_{c}$ have a variance equal to $\sigma_{c}^{\mathrm{Tx}} \sigma_{c}^{\mathrm{Rx}}$, where $\sigma_{c}^{\mathrm{Rx}}$ represents the variance of each path at the receiver side. Once we have selected the set of paths with variances and defined its angles, each MIMO channel realization is performed via a generation of independent random Gaussian variables, with variances defined by the integration method, and at the angle specified by the method. There are different approaches to paths integration; a comparison of the methods for delay and Doppler variables has been presented in (Parra et al., 2003); for the angular variables, we can cite the works of (Pätzold et al., 2008) for several integration methods, while (Pancaldi et al., 2006) explores the method of Gaussian Quadrature Rules (GQR).

An example of this approach is presented in the following figure, where the GQR method has been utilized for the paths integration. At Tx the PAS has uniform shape, while at Rx is Gaussian type. In the figure, it has been also depicted the angles of the paths. An example of this approach will be presented in detail in the following section. 

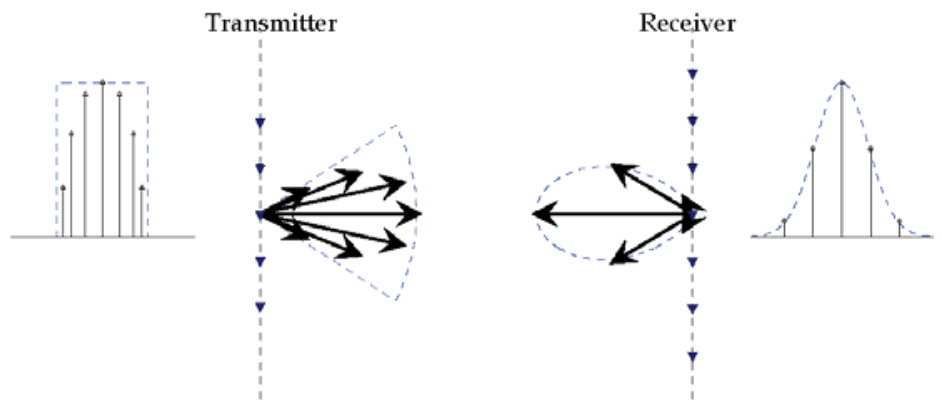

Fig. 11. An example of the method of paths integration.

As can be seen from Fig. 11, the continuous xPAS are approximatted by seven paths at the transmitter and five paths at the receiver, wich leads to an approximation of 35 paths for the entire xPAS.

\subsection{Sum of complex functions}

Under this approach, the approximations in the sense of eq. (31) no longer contain delta functions as paths, but they consist of complex functions. Among the main approaches found in literature, we can first say that all have considered approximations in the way:

$$
h\left(\varphi^{\mathrm{Tx}}, \varphi^{\mathrm{Rx}}\right)=\sum_{c=1}^{c} \beta_{c} \psi_{c}\left(\varphi^{\mathrm{Tx}}\right) \xi_{c}\left(\varphi^{\mathrm{Rx}}\right) .
$$

It means, functions composed via the outer product of artificial paths at the transmitters $\psi_{c}\left(\varphi^{\mathrm{Tx}}\right)$, and artificial paths at the receiver $\xi_{c}\left(\varphi^{\mathrm{Rx}}\right)$. These functions, however, are not defined in the angular domain in the available works, but they are used in the space domain. Then, a channel realization $\mathbf{H}$ can be performed with:

$$
\mathbf{H}=\sum_{c=1}^{c} \beta_{c} \xi_{c} \psi^{T}{ }_{c},
$$

where (columns) vectors $\psi_{c}$ and $\xi_{c}$ belong to the expansion of the spatial channel realizations at the transmitter and receiver sides, respectively. The matrix $\mathbf{H}$ can be stacked in a vector $\mathbf{H}_{v e c}$ through the vec(.) operator, which arranges all columns of the vector in a tall vector. This can be carried out for the representation in (38), with the Kronecker product $(\otimes)$ of the complex functions, as in:

$$
\mathbf{H}_{\text {vec }}=\operatorname{vec}\{\mathbf{H}\}=\sum_{c=1}^{c} \beta_{c} \psi_{c} \otimes \xi_{c} .
$$

The autocorrelation values of the MIMO channel can be represented as a tensor, as in (28), or as a matrix $\mathbf{R}_{\mathbf{H}_{v e c}}$, as follows:

$$
\mathbf{R}_{\mathbf{H}_{v e c}}=E\left\{\mathbf{H}_{v e c} \mathbf{H}_{v e c}{ }^{H}\right\}=\sum_{c=1}^{C} \sigma_{c c}\left(\psi_{c} \otimes \xi_{c}\right)\left(\psi_{c} \otimes \xi_{c}\right)^{H},
$$


where the variance $\sigma_{c c}$ of the weights can be calculated by multiplying $\mathbf{R}_{\mathbf{H}_{v e c}}$ with $\left(\psi_{c} \otimes \xi_{c}\right)^{H}$ at the left, and its complex transpose by the right, as in:

$$
\left(\psi_{c} \otimes \xi_{c}\right)^{H} R_{H_{v c c}}\left(\psi_{c} \otimes \xi_{c}\right)=\sigma_{c c} .
$$

As the vector $\left(\psi_{c} \otimes \xi_{c}\right)^{H}$ is composed of the Kronecker product of two orthogonal functions, doing (41) with two different vectors, $\left(\psi_{c} \otimes \xi_{c}\right)$ and $\left(\psi_{d} \otimes \xi_{d}\right)$ will generate the coupling matrix $\Omega$, formed by entries $\sigma_{c d}$,

$$
\boldsymbol{\Omega} \triangleq\left[\begin{array}{cccc}
\sigma_{11} & \sigma_{12} & \cdots & \sigma_{1, C} \\
\sigma_{21} & \sigma_{22} & \cdots & \sigma_{2, C} \\
\vdots & \vdots & \ddots & \vdots \\
\sigma_{C, 1} & \sigma_{C, 2} & \cdots & \sigma_{C, C}
\end{array}\right] .
$$

Defining $\Psi, \odot, \mathbf{G}$, and $\xi$ as the matrix of vectors of the expansion at the transmitter side, the Walsh-Hadamard operator, a matrix of size $\mathrm{M} \times \mathrm{N}$ with i.i.d complex Gaussian variables and the vectors of the receiver side, respectively. Then, the state of the art channel simulators generate channel realizations with:

$$
\mathbf{H}=\xi(\tilde{\mathbf{\Omega}} \odot \mathbf{G}) \boldsymbol{\Psi}^{T} .
$$

Where $\tilde{\boldsymbol{\Omega}}$ is obtained by performing square root over each of the elements of $\boldsymbol{\Omega}$.

In (Weichselberguer, 2005) the matrices $\boldsymbol{\Psi}$ and $\xi$ are formed by the eigenvectors of $\mathbf{R}^{\mathrm{Tx}}$ and $\mathbf{R}^{\mathrm{Rx}}$, which are the one sided correlations at $\mathrm{Tx}$ and $\mathrm{Rx}$, respectively; where those are calculated with:

$$
\mathbf{R}^{\mathrm{Tx}}=E\left\{\mathbf{H}^{H} \mathbf{H}\right\} ; \quad \mathbf{R}^{\mathrm{Rx}}=E\left\{\mathbf{H H}^{H}\right\} .
$$

In (Sayeed, 2002), the matrices $\Psi$ and $\xi$ are composed of AMVs, obtained from dividing the angles of interest in equally spaced paths. The number of vector in each matrix depends on the selected number of paths.

In (Kontorovich et al., 2008) and (Alcocer-Ochoa et al., 2008), matrices $\boldsymbol{\psi}$ and $\xi$ are composed of the Discrete Prolate Sequences (DPS). These functions can be obtained through the eigen decomposition of a kernel proportional to:

$$
\mathbf{R}^{\mathrm{Tx} / \mathrm{Rx}}=\frac{\sin \left(2 \pi k_{m} d(m-n)\right)}{(m-n) \pi}, \quad m, n=0,1, \ldots, \mathrm{M}-1,
$$

where $k_{m}$ is the maximum wavenumber spread and $d$ is the distance between elements of the array. The choice of DPS is because they are a basis for channel expansion that depends only on the dimension of the antenna array and the maximum wavenumber, which is also directly related to the maximum AoA or AoD. The number of functions does not depend on the shape of the PAS. These functions are also the approximate Eigenfunctions for several profiles defined in standards. This can be understood considering that the kernel in (45) implies that in the WNS, the kernel Prolate should be a square function. This have been plotted in Fig. 12 along with the GM of truncated versions of Gaussian and Von Misses PAS; 
due to their common shapes in the WNS, is not strange that Prolate be a good basis for approximation of channel realizations becoming from the mentioned PAS.

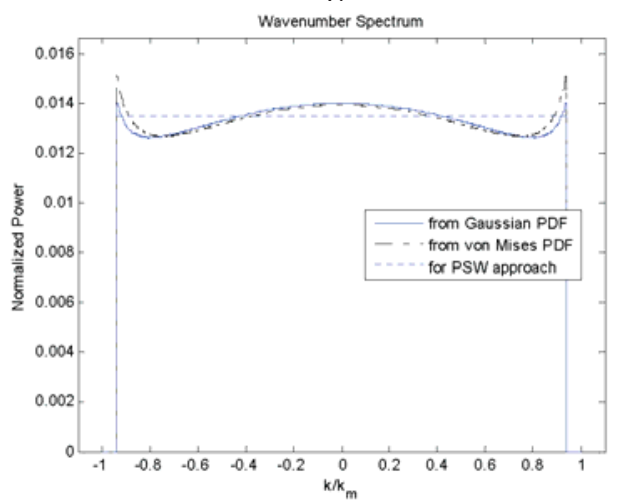

Fig. 12. WNS of the Prolate Kernel and most considered PAS in its truncated versions.

Finally, in Fig. 12 is presented the representation of the complex functions (actually Prolate) in a polar representation. Note that the artificial paths do not have physical sense, but provide an advantage in signal representation.
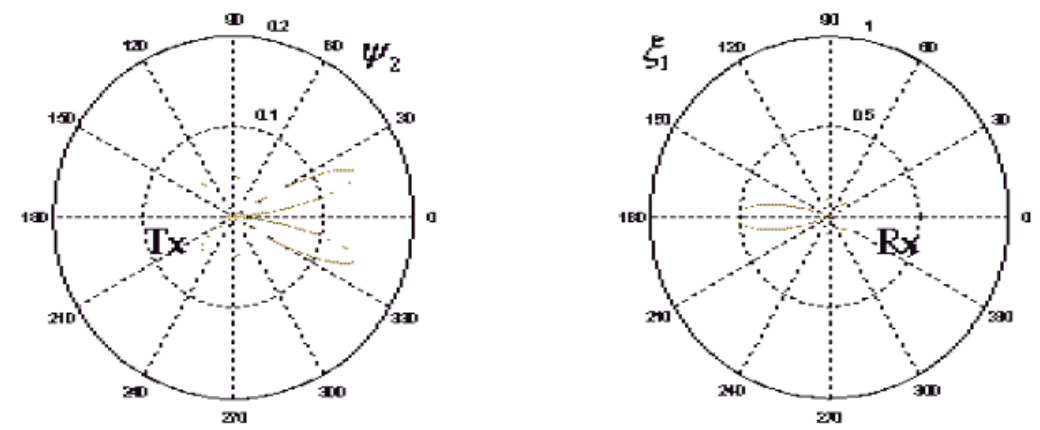

Fig. 13. Example of the complex funtions for the implementation of simulators.

\subsection{A note on state of the art emulators}

In this chapter has been reviewed the topic of channel simulator, but in reality, emulators construction is an important topic as well. When we consider the practical implementation issues of the simulators, several new problems become important that were left aside in simulators. Principally, modern simulators have to deal with different configurations at each side of the links, i.e. sometimes a configuration of a 3x3 MIMO will be necessary, and other times a $4 \times 2$. This imply that the emulator be flexible enough to count with the topologies needed in the newest standards. Emulators also have to be generic enough to provide capacities for different standards, as in the near future, communication systems comprising several standards in the same designs will be mandatory. In other hand, the necessity to increase data rates imposes a problem to real time channel emulators, which have to distort signals in real operation of systems; this is a big issue in coming standards where data 
throughputs are in orders of $\mathrm{GHz}$. It is interesting to note that there are also several technologies for channel emulators, since digital implementations, passing through RF passive and active circuits, up to optical technologies, and all with the same aim.

In (Kontorovich \& Parra-Michel, 2008) can be reviewed some of the principal companies that work in constructing channel emulators, as well as a review of the latest patents in this area, while in (Loyola et al., 2008), an emulator with the idea of artificial paths is presented.

\section{Performance analysis}

One important thing in simulation is to guarantee that the employed method achieves some degree of similarity with the target model. In this context, some scenario defined in terms of certain xPAS will be utilized to provide some target SCF. From this channel, the proposed methods will be executed and its generated SCF will be stored. Comparisons between those two SCF will provide the similarity estimation.

\subsection{A metric for performance comparison}

The metric employed for the comparison is given by the direct distance between two matrices $\mathbf{A}$ and $\mathbf{B}$ specifying two SCF in the sense of the Frobenius norm: $\|\mathbf{A}-\mathbf{B}\|_{F}$, where Frobenius norm is defined as

$$
\|\mathbf{A}\|_{F}=\sqrt{\sum_{i} \sum_{j}\left|\mathbf{A}_{i j}\right|^{2}} .
$$

Translated to our specific case, we will get the existent difference of two SCF; let be $\mathbf{R}^{A}$ and $\mathbf{R}^{B}$ those matrices and they will be compared as follows:

$$
\left\|\mathbf{R}_{N}^{A}-\mathbf{R}_{N}^{B}\right\|_{F},
$$

where for any correlation matrix $\mathbf{R}, \mathbf{R}_{\mathbf{N}}$ is its normalized version, obtained with:

$$
\mathbf{R}_{N}=\frac{\mathbf{R}}{\|\mathbf{R}\|_{F}} \text {. }
$$

It is remarkable that this metric is equivalent to that defined by:

$$
d_{1}\left(\mathbf{R}^{A}, \mathbf{R}^{B}\right)=\frac{\left\|\mathbf{R}_{N}^{A}-\mathbf{R}_{N}^{B}\right\|_{F}}{\left\|\mathbf{R}_{N}^{A}\right\|_{F}\left\|\mathbf{R}_{N}^{B}\right\|_{F}},
$$

where $d_{1}($.$) is the distance between two matrices, considering that \left\|\mathbf{R}_{N}\right\|_{F}=1$. Another equivalent expression for (49) is:

$$
d_{1}\left(\mathbf{R}^{A}, \mathbf{R}^{B}\right)=\frac{\left\|\operatorname{vec}\left(\mathbf{R}_{N}^{A}\right)-\operatorname{vec}\left(\mathbf{R}_{N}^{B}\right)\right\|}{\left\|\operatorname{vec}\left(\mathbf{R}_{N}^{A}\right)\right\|\left\|\operatorname{vec}\left(\mathbf{R}_{N}^{B}\right)\right\|},
$$

which can be interpreted as the normalized distance between correlation matrices, when those are seen as elements of a vector space.

\subsection{Simulation results}

Upon defined a xPAS composed of a uniform shape PAS at Tx and a Gaussian type PAS at $\mathrm{Rx}$, and with the use of metric $d_{1}($.$) , the performance of main simulation methods were$ 
compared. The results are shown in Table 4, where the PAS at Tx was uniform from $-20^{\circ}$ to $20^{\circ}$, while PAS at Rx considered a standard deviation of $7^{\circ}$. The system is a $9 \times 9$ uniform linear array MIMO comunication system, the antenna intespace is of a half of the wavelength of the carrier transmitted signal $(2 \mathrm{GHz})$. In Table 2, VM stands for Weichselberguer method, MEA is the method of paths integration into equal areas, MGQR is the method of GQR.

\begin{tabular}{lll}
\hline \hline Method & No. Fun. (error $<0.1)$ & No. Fun. (error $<0.01)$ \\
\hline WM & 26 & 42 \\
MEA & 110 & 1600 \\
MGQR & 169 & 900 \\
Prolates & 49 & 49 \\
\hline
\end{tabular}

Table 4. Performance comparison of the simulation methods

As the complexity of the methods are related with the number of functions utilized in the approximation, it is clear that the best method is WM, which uses the optimal functions, with the trade off of being dependent on the specific SCF. The methods of paths integration do not behave very well. In the other hand, Prolate are not so far from WM, but they have the remarkable property that are invariant to the specific shape of the XPAS and only depends on the external parameters (maximum wavenumber dispersion and size of the antenna array). This property opens the possibility of Prolate to be a good basis for construction of a generic channel simulator.

\section{Open research problems and Future Trends}

From the concepts reviewed in this Chapter, and with the aid of (Kontorovich \& ParraMichel, 2008), we can state that the topic of channel simulation is far to be closed. This is due to the fact that the communication systems are continuously appearing, demanding new channel models and its efficient simulators. For example, the IEEE 802.15c standard would require the elevation angle for beamforming applications. Moreover, the new standards suggest the use of either OFDM or single carrier schemes, and some mixtures between them to improve the system performance. On the other hand, the use of planar antenna arrays both for $\mathrm{Tx}$ and $\mathrm{Rx}$ is also considered. This imply that the study of the propagation phenomena shall include the elevation angle as well as the azimuth one, but up to now, simulators do not consider this domain. Models that account with all degrees of freedom in the Space-Time channels are still to be done. As open research problems we can mention the conception of tractable models to deal with correlation between space and time variables. It is also a matter of research to understand how is connected the efforts of simulators with actual system performance, i.e. it suffices to guarantee approximations of second order statistics, or higher order are necessary to truly emulate the real channels? Additionally, works on channel simulators open the need for works in implementation issues for emulators. We can state that current work lines are principally devoted to a) Diminish the complexity of the simulator implementations, b) Integrate more statistical channel information or environment description and c) Provide flexible simulators to account for several configurations.

In future developments, we can expect that simulators will incorporate all statistical information for all domains (Doppler, AoA, etc.). Those simulators will show generic 
architectures and means to provide channel realizations in an efficient (not complex, yet accurate) way, and perhaps with reduced information, while being versatile enough to provide any channel configuration array.

\section{Conclusions}

Channel modeling and simulation topics have been presented in this Chapter. We intended to present the framework, the main problems, techniques, theories and results in this research area. Several examples were presented in order to clarify the concepts reviewed, with the aim of providing to the reader tools for understanding and manage the concepts of MIMO channel modeling. A comparison of the simulators performance was presented, and the generic properties of Prolate functions were highlighted. We close this Chapter with the mention that channel modeling and simulation is useful and worthy by itself, as it provides an insight to devise algorithms that mitigate the channel introduced distortions. In this sense, we can anticipate that works for channel compensation based on the ideas of artificial paths discussed here, shall be common in the near future.

\section{Acknowledgment}

This work was supported by research grant Intel CERMIMO-2008 and CONACYT research projects 84559-Y and 53769 .

\section{References}

3GPP (2003). Spatial Channel Model for Multiple Input - Multiple Output (MIMO) Simulations TR 25.996 v6.1.0 Technical Report.

Alcocer-Ochoa, A; Kontorovitch, V. \& Parra-Michel, R. (2008) A Wideband MIMO Channel Model based on Structured Vector Modes using Orthogonalization. Proc. of IEEE WICOM 2008, pp. 1-5. October 2008.

Bello, P. A. Characterization of randomly time-variant linear channels. IEEE Trans. Commun. Syst., Vol. 11, pp. 360-393.

COST 207. (1989). Digital land mobile radio communications. Office for Official Pub. of the European Communities, Final Report. Luxembourg, 1989.

Durgin, G. D. (2003). Space-Time Wireless Channels, Prentice Hall PTR, ISBN: 0-13-065647X, New Jersey, USA, 2003.

Ertel, R. B. \& Reed, J. H. (1999). Angle and Time of Arrival Statistics for Circular and Elliptical Scattering Models, IEEE Journal on Selected Areas in Communications. Vol. 17, No. 11, pp. 1829-1840, November 1999.

Fechtel, S. A. (1993). A novel approach to modeling and efficient simulation of frequency selective radio channels. IEEE J. Sel. Areas Commun. Vol. 11, pp. 422-431, 1993.

Fuhl, J.; Molisch, A. F. \& Bonek, E. (1998). Unified Channel Model for Mobile Radio Systems with Smart Antennas, IEE Procedures on Radar and Sonar Navigation, Vol. 145, No. 1, pp. 32-41. February 1998.

Gans, M. J. (1972). A Power-Spectral Theory of Propagation in the Mobile-Radio Environment. IEEE Trans. on Vehic. Tech., Vol. 21, No. 1, pp. 27-38. February 1972. 
Kennedy, R. S. (1969). Fading Dispersive Communication Channels, Wiley-Interscience, ISBN: 471-46903-3, USA.

Kontorovich, V. \& Parra-Michel, R. (2008). Review of Patents in Simulation of Broadband Communication Channels. Journal of Recent Patents on Electrical Engineering, Vol. 1, pp. 188-200, 2008.

Kontorovich, V.; Primak, S.; Alcocer-Ochoa, A. \& Parra-Michel, R. (2008). MIMO Channel Orthogonalisations applying Universal Eigenbasis. IET Signal Processing, Vol. 2, No. 2, pp. 87-96, 2008.

Laurila, J.; Molisch, A. F. \& Bonek, E. (1998). Influence of the Scatters Distribution on the Power Delay Profile and the Power Azimuth Spectra of Mobile Radio Channels. IEEE Intl. Symp. on Spread Spectrum Tech. and Applications. Vol. 1, pp. 267-271, Sept. 1998.

Loyola. I. J.; Alcocer-Ochoa, A; Parra-Michel, R; Kontorovich, V. \& Orozco-Lugo, A. (2008) An Efficient wideband channel emulator based on orthogonal functions expansion. Proc. of IEEE WICOM 2008, pp. 1-5, October 2008.

Molisch, A. F. (2004). A Generic Model for MIMO Wireless Propagation Channels in Macroand Microcells. IEEE Trans. on Signal Processing, Vol. 52, No. 1, pp. 61-71, January 2004.

Molisch, A. F.; Asplund, H.; Heddergott, R.; Steinbauer, M. \& Zwick, T. (2006). The COST259 Directional Channel Model-Part I: Overview and Methodology. IEEE Transactions on Wireless Communications, Vol. 5, No. 12, pp. 3421-3433. December 2006.

Pancaldi, F.; Greco, P. \& Vitetta, G. M. (2006). GQR-Based models for directional wireless channels. IEEE Trans. on Wireless Commun., Vol. 5, No. 3, pp. 642-651. March 2006.

Papoulis, A \& Pillai, S. U. (2002). Probability, Random Variables, and Stochastic Processes. Mc Graw Hill. 4th Edition, New York, 2002

Parra-Michel, R.; Kontorovitch, V.; Orozco-Lugo, A. G.; Lara, M. (2003). Computational Complexity of Narrowband and Wideband Channel Simulators. IEEE Vehicular Technology Conference, VTC 2003 -Fall, October 2003.

Parsons, J. D. (2000). The mobile radio propagation channel, $2^{\text {nd }}$ Edition, John Wiley \& Sons, ISBN: 0-471-98857-X, England.

Pätzold, M. (2002). Mobile fading channels, John Wiley \& Sons, 2002.

Pätzold, M.; Hogstad, B. O. \& Youssef, N. (2008). Modeling, Analysis and Simulation of MIMO Mobile-to-Mobile Fading Channels. IEEE Transactions on Wireless Communications, Vol. 7, No. 2, pp. 510-520. February 2008.

Petrus, P; Reed, J. H. \& Rappaport, T. S. (2002). Geometrical-Based Statistical Macrocell Channel Model for Mobile Environments, IEEE Transactions on Communications, Vol. 50, No. 3, pp. 495-502. March 2002.

Piechocki, R. J.; McGeehan, J. P. \& Tsoulos, G. V. (2001). A New Stochastic Spatio-Temporal Propagation Model (SSTPM) for Mobile Communications with Antenna Arrays, IEEE Transactions on Communications, Vol. 49, No. 5, pp. 855-862, May 2001.

Salz, J. \& Winters, J. H. (1994). Effect of Fading Correlation on Adaptive Arrays in Digital Mobile Radio. IEEE Trans. on Vehic. Tech., Vol. 43, No. 4, pp. 1049-1057. November 1994.

Sayeed, A. M. (2002). Deconstructing Multiantenna Fading Channels. IEEE Transactions on Signal Processing. Vol. 50, No. 10, pp. 2563-2579, October 2002. 
Steinbauer, M.; Molish, A. F. \& Bonek, E. (2001). The double directional channel. IEEE Antennas Propag. Mag., Vol. 43, pp. 52-63. Aug. 2001.

van Trees, H. L. (2002). Detection, Estimation and Modulation Theory, Part IV. John Wiley E Sons, 2002.

Weichselberger, W. (2003). Spatial Structure of Multiple Antenna Radio Channels, Ph. D. Thesis, December 2003, Wien.

Weichselberger, W.; Herdin, H.; Özcelik, H. \& Bonek, E. (2006). A Stochastic MIMO Channel Model with Joint Correlation of Both Link Ends, IEEE Transactions on Wireless Communications, Vol. 5, No. 1, pp 90-100, January 2006.

Woerner B. D. ; Reed, J. H. \& Rappaport, T. S. (1994). Simulation issues for future wireless modems. IEEE Commun. Mag., Vol. 32, No. 7, pp. 42-53. 


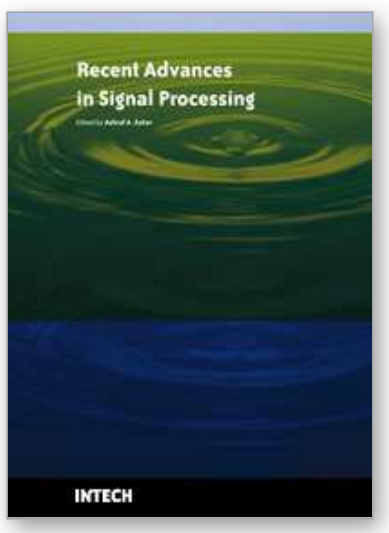

\author{
Recent Advances in Signal Processing \\ Edited by Ashraf A Zaher
}

ISBN 978-953-307-002-5

Hard cover, 544 pages

Publisher InTech

Published online 01, November, 2009

Published in print edition November, 2009

The signal processing task is a very critical issue in the majority of new technological inventions and challenges in a variety of applications in both science and engineering fields. Classical signal processing techniques have largely worked with mathematical models that are linear, local, stationary, and Gaussian. They have always favored closed-form tractability over real-world accuracy. These constraints were imposed by the lack of powerful computing tools. During the last few decades, signal processing theories, developments, and applications have matured rapidly and now include tools from many areas of mathematics, computer science, physics, and engineering. This book is targeted primarily toward both students and researchers who want to be exposed to a wide variety of signal processing techniques and algorithms. It includes 27 chapters that can be categorized into five different areas depending on the application at hand. These five categories are ordered to address image processing, speech processing, communication systems, time-series analysis, and educational packages respectively. The book has the advantage of providing a collection of applications that are completely independent and self-contained; thus, the interested reader can choose any chapter and skip to another without losing continuity.

\title{
How to reference
}

In order to correctly reference this scholarly work, feel free to copy and paste the following:

R. Parra-Michel, A. Alcocer-Ochoa, A. Sanchez-Hernandez and Valeri Kontorovich (2009). MIMO Channel Modeling and Simulation, Recent Advances in Signal Processing, Ashraf A Zaher (Ed.), ISBN: 978-953-307002-5, InTech, Available from: http://www.intechopen.com/books/recent-advances-in-signal-processing/mimochannel-modeling-and-simulation

\section{INTECH}

open science | open minds

\section{InTech Europe}

University Campus STeP Ri

Slavka Krautzeka 83/A

51000 Rijeka, Croatia

Phone: +385 (51) 770447

Fax: +385 (51) 686166

www.intechopen.com

\section{InTech China}

Unit 405, Office Block, Hotel Equatorial Shanghai

No.65, Yan An Road (West), Shanghai, 200040, China 中国上海市延安西路65号上海国际贵都大饭店办公楼 405 单元 Phone: +86-21-62489820

Fax: $+86-21-62489821$ 
(C) 2009 The Author(s). Licensee IntechOpen. This chapter is distributed under the terms of the Creative Commons Attribution-NonCommercial-ShareAlike-3.0 License, which permits use, distribution and reproduction for non-commercial purposes, provided the original is properly cited and derivative works building on this content are distributed under the same license. 